\title{
A!
}

This is an electronic reprint of the original article.

This reprint may differ from the original in pagination and typographic detail.

Mao, Dong; Feng, Tianxian; Zhang, Wending; Lu, Hua; Jiang, Yajun; Li, Peng; Jiang, Biqiang;

Sun, Zhipei; Zhao, Jianlin

Ultrafast all-fiber based cylindrical-vector beam laser

Published in:

Applied Physics Letters

DOI:

$10.1063 / 1.4973922$

Published: 09/01/2017

Document Version

Publisher's PDF, also known as Version of record

Please cite the original version:

Mao, D., Feng, T., Zhang, W., Lu, H., Jiang, Y., Li, P., Jiang, B., Sun, Z., \& Zhao, J. (2017). Ultrafast all-fiber based cylindrical-vector beam laser. Applied Physics Letters, 110(2), [021107].

https://doi.org/10.1063/1.4973922

This material is protected by copyright and other intellectual property rights, and duplication or sale of all or part of any of the repository collections is not permitted, except that material may be duplicated by you for your research use or educational purposes in electronic or print form. You must obtain permission for any other use. Electronic or print copies may not be offered, whether for sale or otherwise to anyone who is not an authorised user. 


\section{Ultrafast all-fiber based cylindrical-vector beam laser}

Dong Mao, Tianxian Feng, Wending Zhang, Hua Lu, Yajun Jiang, Peng Li, Biqiang Jiang, Zhipei Sun, and Jianlin Zhao

Citation: Appl. Phys. Lett. 110, 021107 (2017); doi: 10.1063/1.4973922

View online: https://doi.org/10.1063/1.4973922

View Table of Contents: http://aip.scitation.org/toc/apl/110/2

Published by the American Institute of Physics

\section{Articles you may be interested in}

Resonance efficiency enhancement for cylindrical vector fiber laser with optically induced long period grating Applied Physics Letters 110, 161104 (2017); 10.1063/1.4980129

Squeezing-enhanced fiber Mach-Zehnder interferometer for low-frequency phase measurement Applied Physics Letters 110, 021106 (2017); 10.1063/1.4973895

Superconductive PT-symmetry phase transition in metasurfaces Applied Physics Letters 110, 021104 (2017); 10.1063/1.4973769

Controlling surface plasmon polaritons at femtosecond timescales on an aluminum-coated grating Applied Physics Letters 110, 021105 (2017); 10.1063/1.4973860

Enhancing light transmission through a disordered waveguide with inhomogeneous scattering and loss Applied Physics Letters 110, 021103 (2017); 10.1063/1.4973459

Engineering topological edge states in two dimensional magnetic photonic crystal Applied Physics Letters 110, 021109 (2017); 10.1063/1.4973990

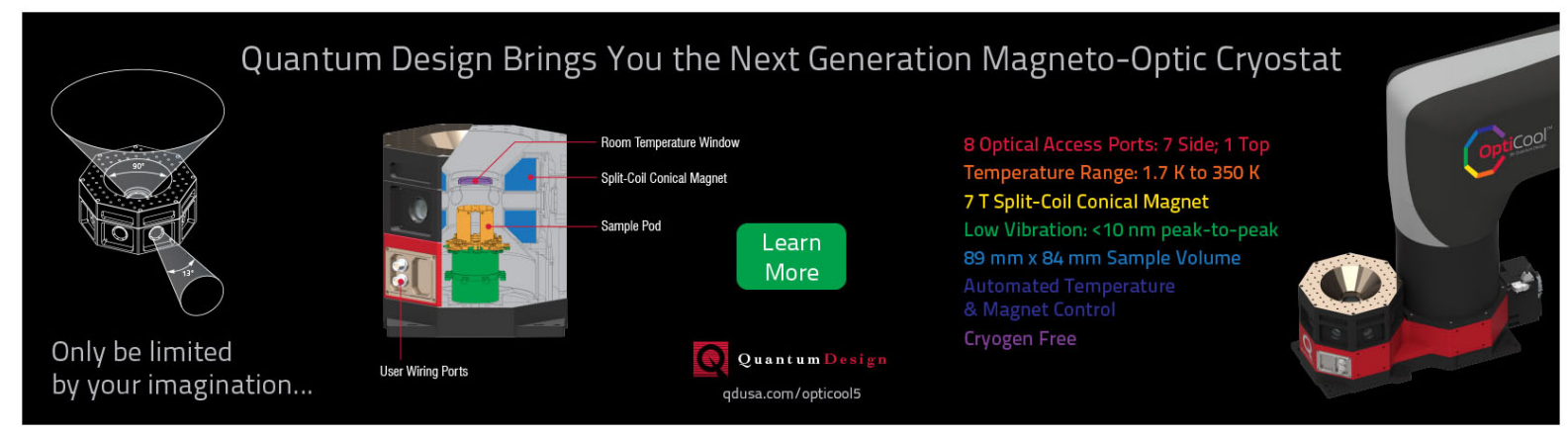




\title{
Ultrafast all-fiber based cylindrical-vector beam laser
}

\author{
Dong Mao, ${ }^{1, a)}$ Tianxian Feng, ${ }^{1}$ Wending Zhang, ${ }^{1}$ Hua Lu, ${ }^{1}$ Yajun Jiang, ${ }^{1}$ Peng Li, ${ }^{1}$ \\ Biqiang Jiang, ${ }^{1}$ Zhipei Sun, ${ }^{2}$ and Jianlin Zhao ${ }^{1}$ \\ ${ }^{1}$ MOE Key Laboratory of Space Applied Physics and Chemistry; Shaanxi Key Laboratory of Optical \\ Information Technology, School of Science, Northwestern Polytechnical University, Xi' an 710072, China \\ ${ }^{2}$ Department of Micro- and Nanosciences, Aalto University, Tietotie 3, FI-00076 Espoo, Finland
}

(Received 17 November 2016; accepted 29 December 2016; published online 10 January 2017)

Cylindrical-vector beams (CVBs) with axial symmetry in polarization and field intensity are gathering increasing attention from fundamental research to practical applications. However, a majority of the CVBs are generated by modulating light beams in free space, and the temporal durations are far away from the ultrafast regime. Here, an ultrafast all-fiber based CVB laser is demonstrated via intermodal coupling in two mode fibers. In the temporal domain, chirp-free pulses are formed with combined actions of the ultrafast saturable absorption, self-phase modulation, and anomalous dispersion. In the spatial domain, the lateral offset splicing technique and a two mode fiber Bragg grating are adopted to excite and extract CVBs, respectively. The ultrafast CVB has an annular profile with a duration of $6.87 \mathrm{ps}$ and a fundamental repetition rate of $13.16 \mathrm{MHz}$, and the output polarization status is switchable between radially and azimuthally polarized states. This allfiber-based ultrafast CVB laser is a simple, low-cost source for diversified applications of nanoparticle manipulation, high-resolution imaging, material processing, spatiotemporal nonlinear optics, etc. Published by AIP Publishing. [http://dx.doi.org/10.1063/1.4973922]

Laser beams with axial symmetry in both polarization and field intensity, the so-called cylindrical-vector beams (CVBs), are gathering increasing attention from fundamental research to practical applications. ${ }^{1-4}$ According to the spatial distribution of the polarization, CVBs are classified as radially polarized, azimuthally polarized, and hybridly polarized beams. Particularly, radially polarized beams can be focused into tighter focal spots with a strong longitudinal field component $^{5}$ and were applied in high-resolution imaging, ${ }^{6}$ nanoparticle manipulation, ${ }^{7}$ material processing, ${ }^{8}$ plasmonic focusing, ${ }^{9}$ and Z-scan technique, ${ }^{10}$ etc. Various techniques have been developed to generate CVBs or vortex beams, such as axial birefringent components, spatial light modulators, angular gratings, and interferometric methods. ${ }^{1-19}$ Very recently, by controlling the geometric phase in a solidstate laser, Forbes et al. demonstrated the direct generation of higher-order Poincaré sphere beams including vector vortex beams and CVBs. ${ }^{20}$ Depending on whether gain media is used, these methods are categorized as passive (extra-cavity) ${ }^{1-15}$ and active (intra-cavity). ${ }^{16-18,20}$ However, in free space, the generation system is rather complicate and tricky, and researchers need to overcome problems like insertion loss and assembling issue in applications of fiber-based communications, sensors, and lasers.

Currently, the generation of CVBs based on few mode fiber or specially designed fiber attracts increasing interest because these systems possess features of excellent flexibility, robust mode confinement, and compact structure. ${ }^{21-24}$ For a two mode fiber (TMF), it guides the fundamental mode $\mathrm{LP}_{01}$ $\left(\mathrm{HE}_{11}\right)$ and the first group higher-order modes $\mathrm{LP}_{11}\left(\mathrm{TE}_{01}\right.$, $\mathrm{TM}_{01}$, and $\mathrm{HE}_{21}$ ) in the scalar (vector) approximation. As the

\footnotetext{
a) Author to whom correspondence should be addressed. Electronic mail: maodong@nwpu.edu.cn
}

eigenmodes $\mathrm{TM}_{01}$ and $\mathrm{TE}_{01}$ are radially and azimuthally polarized beams, respectively, ${ }^{25,26}$ CVBs can be obtained via exciting these modes in TMFs. By offset splicing single-mode fiber (SMF) with TMF or imposing an acoustic flexural wave on TMF to realize the intermodal coupling, continuous, ${ }^{26-28}$ microseconds, ${ }^{29}$ and nanoseconds, ${ }^{30} \mathrm{CVBs}$ were delivered in TMFs or TMF-based lasers. The durations of these CVBs are larger than several nanoseconds, and the ultrafast CVB laser has not been realized. Therefore, it is quite crucial to develop low-cost, all-fiber based ultrafast CVB lasers, especially for the application of spatiotemporal nonlinear optics. ${ }^{31}$

In this paper, we demonstrate an ultrafast all-fiber based CVB laser via intermodal coupling in TMFs. A carbon nanotube saturable absorber (CNT-SA) sharpens the pulse into several picoseconds, while a lateral offset splicing technique and a two mode fiber Bragg grating (TM-FBG) excite and extract CVBs, respectively. The output CVB has an annular intensity profile with a duration of $6.87 \mathrm{ps}$ and a repetition rate of 13.16 MHz, respectively. The proposed laser has an all-fiber configuration, and the operation status is switchable between radially and azimuthally polarized states, providing a costeffective ultrafast CVB source for practical applications.

The formation of the ultrafast CVB is based on combined functions of the mode-locking technique and transverse mode excitation/selection in TMFs. The laser resonator, CNT-SA, offset splicing spot (OSS), and TM-FBG are four key elements to generate ultrafast CVBs. As illustrated in Figure 1, the laser beam sequentially passes through a wavelength division multiplexer (WDM), a 6-m erbium-doped fiber (EDF) (Nufen: EDFC-980-HP), a 3-port circulator, a lateral OSS, and then the laser is reflected by a TM-FBG and propagates through a CNT-SA and an output coupler (OC). A $980 \mathrm{~nm}$ LD pumps the EDF with the WDM. The total length of the SMF and TMF is $8.6 \mathrm{~m}$ and $0.3 \mathrm{~m}$, respectively. The CVB is 


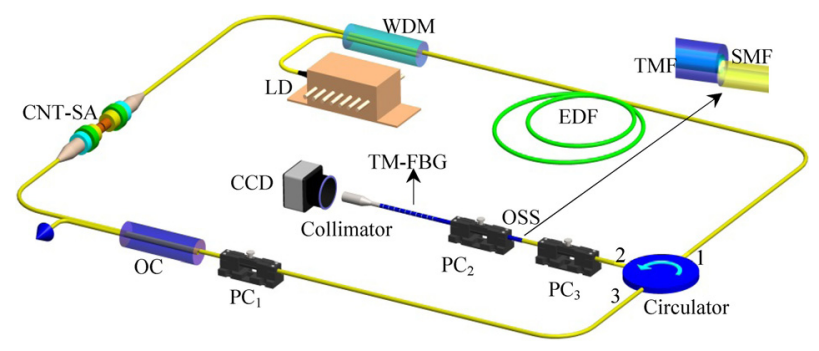

FIG. 1. Setup of the ultrafast CVB fiber laser. LD: laser diode; WDM: wavelength division multiplexer; EDF: erbium-doped fiber; OSS: offset splicing spot; PC: polarization controller; OC: output coupler; CNT-SA: carbon nanotube saturable absorber; TM-FBG: two mode fiber Bragg grating; CCD: charge coupled device. The CNT-SA and the TM-FBG act as the modelocker and transverse mode selector, respectively.

exported through a collimator connected to the end of the TM-FBG, and the transverse distribution of the CVBs is recorded by an infrared charge coupled device (CCD). The temporal behaviors of the CVB are measured from the OC.

An ultrafast CNT-SA is used to start and maintain the mode-locking operation. It is prepared by mixing CNTs with polyvinyl alcohol solution, and then evaporating them in a
Petri dish, similar to the previous reports. ${ }^{32-34}$ The side profile of the CNT film is shown in Figure 2(a), which gives a thickness of $6.86 \mu \mathrm{m}$. The fiber-based SA is formed by sandwiching the CNT film between two facets of a fiber connector, and the nonlinear absorption property of the SA is measured with a balanced twin-detector technique. ${ }^{35}$ As demonstrated in Figure 2(b), the optical transmission increases with the intensity of the illuminating pulse and becomes saturated at a certain value, which is the representative property of SAs. ${ }^{36}$ Through fitting the result with a common model, ${ }^{37}$ the saturable intensity and modulation depth of the SA are given as $8.6 \mathrm{MW} / \mathrm{cm}^{2}$ and $3.3 \%$, respectively.

The offset splicing technique is used to realize intermodal coupling between the fundamental mode and the first group higher-order modes, as illustrated in Figure 3(a). Radially or azimuthally polarized modes can be excited when the two fibers are shifted in the transverse direction, while only the fundamental mode is visualized when the two fiber axes are aligned. ${ }^{21,25}$ The coupling efficiency of the fundamental mode to $\mathrm{TM}_{01}\left(\mathrm{TE}_{01}\right)$ can be obtained through the overlap between the electric field distributions of guided modes in the SMF and TMF, which can be described as ${ }^{38}$

$$
\gamma_{c}=\left[\frac{4 n_{e f f, 1} n_{e f f, 2}}{\left(n_{e f f, 1}+n_{e f f, 2}\right)^{2}}\right]\left|\frac{\iint_{\infty} E_{1}(x, y) E_{2}^{*}(x, y) d x d y}{\sqrt{\iint_{\infty} E_{1}(x, y) E_{1}^{*}(x, y) d x d y \times \iint_{\infty} E_{2}(x, y) E_{2}^{*}(x, y) d x d y}}\right|
$$

where $E_{1}(x, y)$ and $E_{2}(x, y)$ are the electric fields of the guided modes in the SMF and TMF, respectively. $n_{\text {eff }, 1}$ and $n_{\text {eff }, 2}$ are the corresponding effective refractive indices, respectively. Figure 3(b) shows that the coupling efficiency between the $\mathrm{TM}_{01}$ mode and fundamental mode dramatically increases with an increase in the dislocation distance $\Delta R$ when $\Delta R<5.2 \mu \mathrm{m}$. As $\Delta R$ approaches to $5.2 \mu \mathrm{m}$, the electric field of the $\mathrm{TM}_{01}$ mode strongly overlaps with that of the fundamental mode and there exists a maximal coupling efficiency of $20.7 \%$. When $\Delta R$ increases further, the coupling efficiency decreases with gradually subsiding overlap of the electric fields. Figure 3(c) shows the coupling behavior of the $\mathrm{TE}_{01}$ mode at different $\Delta R$, which is similar to that of $\mathrm{TM}_{01}$.
The finite element calculation results show that the $\mathrm{TM}_{01}\left(\mathrm{TE}_{01}\right)$ mode is excited when the polarization of the fundamental mode is parallel (perpendicular) to the dislocation direction. The corresponding intensity profiles and polarizations of $\mathrm{TM}_{01}$ and $\mathrm{TE}_{01}$ modes are shown in the inset of Figures 3(b) and 3(c), respectively. This behavior could be explained by noting that the tangential electric field is continuous across the boundary of two fibers. Thus, by adjusting the orientation between the input polarization and the dislocation direction, the CVBs can be switched between radially and azimuthally polarized states.

Based on the aforementioned analyses, we use an optical fiber fusion splicer to realize the lateral misalignment between the SMF (Corning: SMF-28e) and TMF (OFS: Two
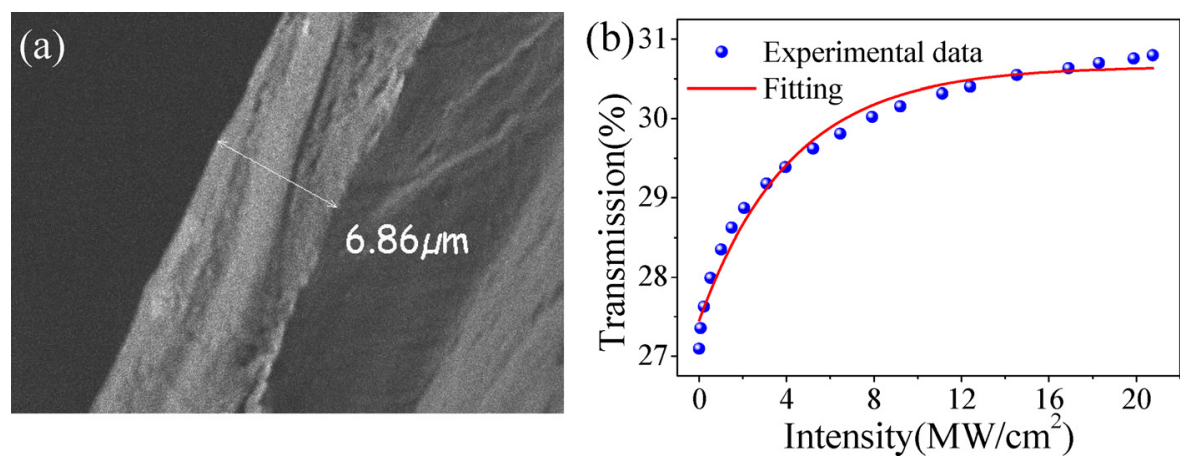

FIG. 2. (a) Side profile of the CNTpolyvinyl alcohol film. (b) Saturable absorption of the CNT-SA. 
(a)
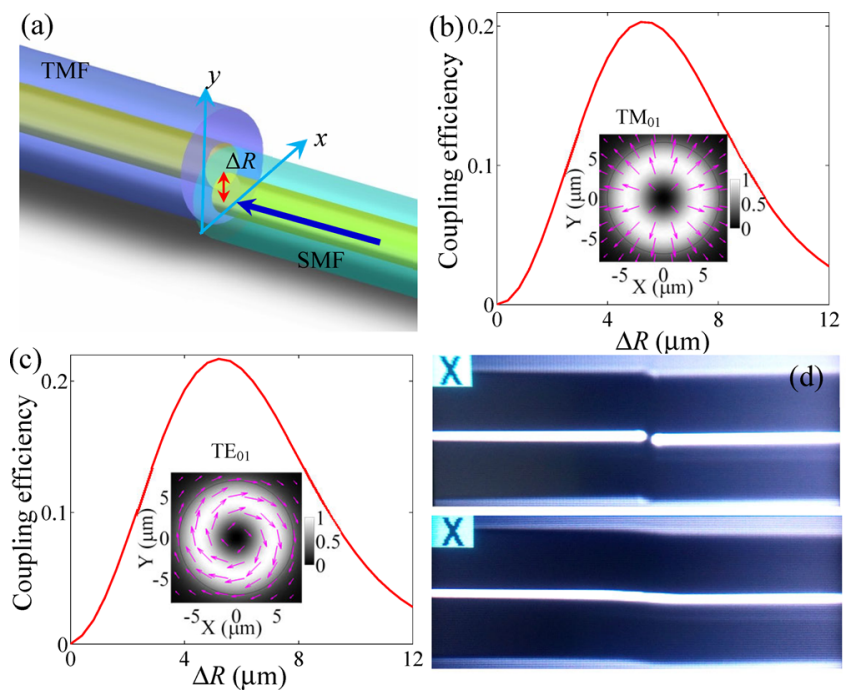

FIG. 3. (a) Sketch map of offset splicing SMF and TMF. Coupling efficiency of the fundamental mode to $\mathrm{TM}_{01}$ mode (b) and $\mathrm{TE}_{01}$ mode (c) at different dislocation distances $\Delta R$. The polarization of the fundamental mode is parallel (b) and perpendicular (c) to the dislocation direction. The insets show the normalized intensity and polarizations of $\mathrm{TM}_{01}$ and $\mathrm{TE}_{01}$ modes, respectively. (d) The micrographs of the OSS before and after splicing the SMF and the TMF.

mode step-index fiber), which allows the positioning and the setting of two fibers in a simple, precise, and reproducible manner. The TMF has a core radius of $7 \mu \mathrm{m}$, a cladding radius of $62.5 \mu \mathrm{m}$, and a step index difference of 0.005 , respectively. The normalized frequency number of the TMF is 3.42 at $1550 \mathrm{~nm}$; thus, only the fundamental mode and the first group higher-order vector modes can be guided in the fiber core. When an optimized lateral misalignment is found, the two fibers are fusion spliced together. The micrographs of two fibers before and after the splicing are illustrated in Figure 3(d).

After the OSS, the fundamental mode and first group higher-order modes coexist in the TMFs. A TM-FBG, fabricated by exposing the TMF to a femtosecond Ti: sapphire laser, is used to select the CVBs. As depicted by the black curve in Figure 4(a), the TM-FBG has three reflection peaks, in which peak 1 represents the 1 st to 1 st order mode reflection, peak 2 represents the 1 st to 2 nd order mode reflection, and peak 3 represents the 2 nd to 2 nd order mode reflection. ${ }^{39}$ Thus, the TM-FBG reflects back the fundamental mode while exports the CVBs when the laser spectrum locates at peak 1 (1st to 1 st order mode reflection). However, when the laser wavelength goes beyond peak 1 , both the fundamental mode and the CVBs pass through the TM-FBG, and the mode purity will be decreased.

At the laser pump of $20 \mathrm{~mW}$, a stable continuous laser is first obtained in the proposed fiber laser. Further tuning the laser pump to $75 \mathrm{~mW}$, the self-start passive mode-locking state can be established. Herein, the spatial distributions of the laser could be the fundamental mode, the first group higher-order vector modes, or the combination of them. Further adjusting the PC before the OSS, azimuthally or radially polarized CVBs can be obtained, and the mode-locking state is still maintained. The temporal behaviors of the ultrafast CVB at the pump power of $80 \mathrm{~mW}$ are plotted in Figure 4. As depicted by the blue curve in Figure 4(a), the optical spectrum of the mode-locked pulse has a smooth profile without any spectral sidebands, which is caused by the spectral filtering of TM-FBG. ${ }^{34,40}$ The central wavelength and 3$\mathrm{dB}$ bandwidth are $1550.5 \mathrm{~nm}$ and $0.34 \mathrm{~nm}$, respectively. Note that the pulse spectrum is slightly broader than the reflection of the TM-FBG, which affects the mode purity of the CVB, and will be discussed in detail in the following paragraphs. As described in Figure 4(b), the pulse duration is $6.87 \mathrm{ps}$ using a Sech ${ }^{2}$ fitting, which is calculated from the autocorrelation width of $10.5 \mathrm{ps}$. The time-bandwidth product of the laser is estimated as 0.31 , suggesting that the pulse is a chirp-free soliton. The radio frequency spectrum in the inset of Figure 4(b) demonstrates that the fundamental repetition rate of the pulse is $13.6 \mathrm{MHz}$. The signal-to-noise ratio is larger than $60 \mathrm{~dB}$, confirming the high stability of the output pulses. The pulse-pulse interval is measured as $76 \mathrm{~ns}$ from
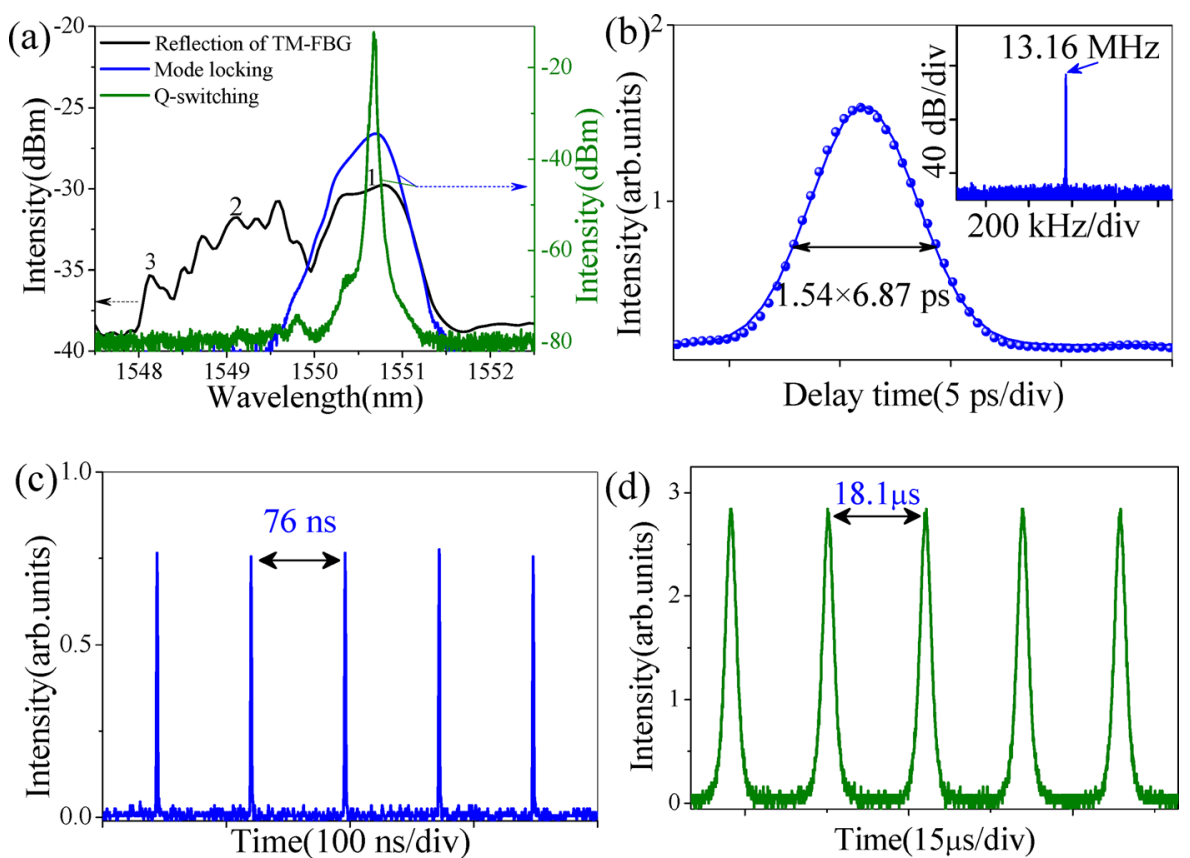

FIG. 4. (a) Reflection of the TM-FBG, and spectra of mode-locked and Qswitched pulses. (b) Autocorrelation trace of the mode-locked pulse, the inset is the radio frequency spectrum. (c) Mode-locked and (d) Q-switched pulse trains. 

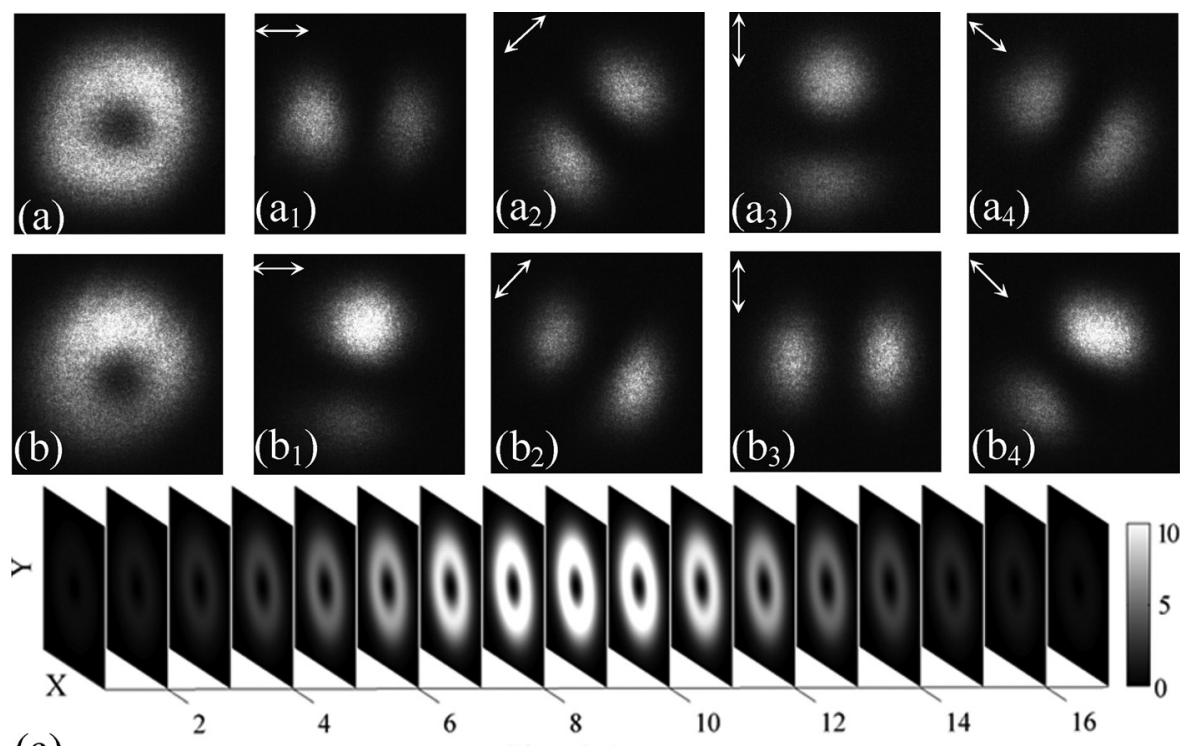

(c)

2

4

6

Time (ps)

10

12

14

16
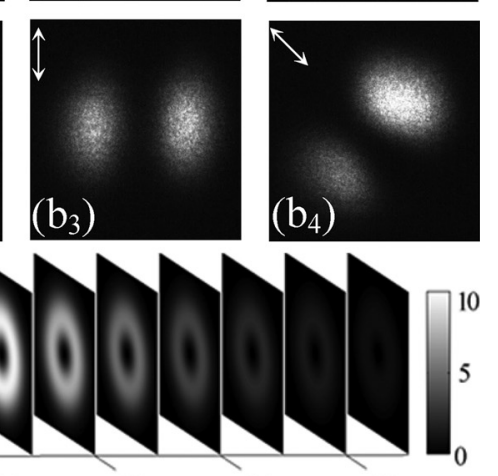

Figure 4(c), which is equal to the round-trip time of the fiber laser. Based on these results, we can confirm that the mode-locked single-pulse is formed in the fiber laser. The Q-switched CVBs are also given for comparisons and analyses, as depicted by the olive-coloured curve in Figures 4(a) and 4(d).

The spatial distribution of the beam profile is shown in Figure 5(a), which has an annular intensity profile with a dark spot at the center. The polarization state of the beam is analyzed by placing a polarizer in front of the CCD. After passing through the linear polarizer at four different orientations, the intensity distributions are shown in Figures $5\left(a_{1}\right)-5\left(a_{4}\right)$, respectively. The direction of the passed light rotates with the transmission axis of the polarizer, and the dark bands are always perpendicular to transmission axis of the polarizer, indicating that the output laser beam is radially polarized. ${ }^{27}$ An azimuthally polarized ultrafast CVB can also be achieved by tuning the PC before the OSS, as shown in Figure 5(b). It is demonstrated that the mode-locked fiber laser can deliver switchable radially or azimuthally polarized ultrafast CVBs. Three-dimensional images of the ultrafast CVBs are plotted to clearly understand the experimental results. As illustrated in Figure 5(c), the profile and polarization of the CVB keep unchanged in the transverse plane. However, the real intensity of the CVB changes with the time following a $\mathrm{Sech}^{2}$ function. Considering a transverse mode area of $158 \mu \mathrm{m}^{2}$ in TMF and a duration of $6.87 \mathrm{ps}$, the ultrafast CVB can be regarded as a hollow tubular beam with a length of $1410 \mu \mathrm{m}$ viewed in the three-dimensional space.

This mode-locked CVB operation can be transformed into a Q-switched state by pressing or bending the SMF near the $\mathrm{PC}_{1}$. Such transformation between two states is attributed to the press- or bend-induced loss on the fiber laser. For example, we find that a smaller (bigger) loss usually corresponds to a mode-locked (Q-switched) operation. The olivecoloured curves in Figures 4(a) and 4(d) show the optical spectrum and pulse train at a pump power of $141 \mathrm{~mW}$, which give the spectral bandwidth of $0.03 \mathrm{~nm}$, the pulse interval of $18.1 \mu \mathrm{s}$, and the pulse duration of $2.38 \mu \mathrm{s}$, respectively. The spatial intensity distributions of the Q-switched CVBs are shown in Figure 6. One can find that the intensity contrast of the CVB in Figure 6 is better than that of Figure 5, for example, the central singularity of the CVB in Figure 6(a) is clearer than that of Figure 5(a). Moreover, in the azimuthal direction, the field distribution of the CVB in Figure 6(a) is more uniform than that of Figure 5(a). Consequently, we infer that the purity of the Q-switched CVB is better than that of the mode-locked CVB, which can be attributed to the different spectral bandwidths of two pulses. As depicted in Figure 4(a), the optical spectrum of the Q-switched pulse is quite narrow and locates at the center of the reflection spectrum of the TM-FBG. In this case, most of the linearly polarized fundamental modes is reflected back into the fiber resonator, and high-purity CVBs are exported from the
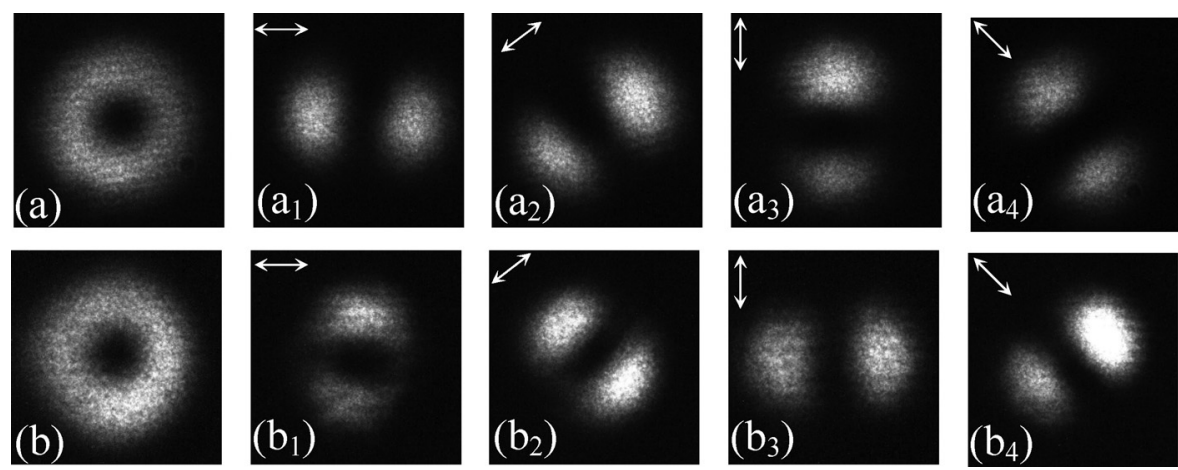

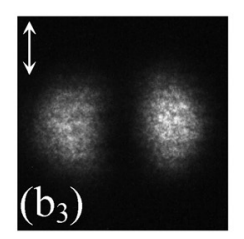

FIG. 6. Intensity distributions of the Q-switched CVBs. (a) Radially and (b) azimuthally polarized CVBs before and after passing a polarizer whose transmission orientation is represented by arrows. 
cavity. However, the spectrum of mode-locked pulses is broader than the reflection spectrum of the TM-FBG. As a result, the fundamental mode that goes beyond the reflection spectrum leaks from the TM-FBG, and the purity of the mode-locked CVB is decreased due to the influence of the residual fundamental mode.

In conclusion, we demonstrate a mode-locked all-fiber laser allowing the direct generation of ultrafast CVBs via the mode conversion and selection with an OSS and TM-FBG, respectively. The radially polarized $\mathrm{CVB}$ has a temporal duration of $6.87 \mathrm{ps}$ and a spectral bandwidth of $0.34 \mathrm{~nm}$, respectively. By tuning the $\mathrm{PC}$, the operation can be switched between the radially and azimuthally polarized states. It is indicated that pulses with cylindrical symmetry in polarization can exist in the TMF lasers, different from that of standard solitons with homogenous polarization states in the SMF lasers. The ultrafast CVBs exhibit unique features in both the temporal and spatial domains, providing a unique light source for spatiotemporal nonlinear optics, laser machining, nanoparticle manipulation, and spatial modedivision multiplexed systems. ${ }^{41}$

This work was supported by the National Natural Science Foundation of China (Grant Nos. 61575162, 11634010, 61405161, 61675169, 61505165, and 11404262).

${ }^{1}$ Q. Zhan, Adv. Opt. Photonics 1(1), 1-57 (2009).

${ }^{2}$ G. M. Lerman, A. Yanai, and U. Levy, Nano Lett. 9(5), 2139-2143 (2009).

${ }^{3}$ P. Anger, P. Bharadwaj, and L. Novotny, Phys. Rev. Lett. 96(11), 113002 (2006).

${ }^{4}$ X. L. Wang, J. Chen, Y. Li, J. Ding, C. S. Guo, and H. T. Wang, Phys. Rev. Lett. 105(25), 253602 (2010).

${ }^{5}$ R. Dorn, S. Quabis, and G. Leuchs, Phys. Rev. Lett. 91(23), 233901 (2003).

${ }^{6}$ X. Xie, Y. Chen, K. Yang, and J. Zhou, Phys. Rev. Lett. 113(26), 263901 (2014).

${ }^{7}$ H. Wang, L. Shi, B. Lukyanchuk, C. Sheppard, and C. T. Chong, Nat. Photonics 2(8), 501-505 (2008).

${ }^{8}$ M. Meier, V. Romano, and T. Feurer, Appl. Phys. A 86(3), 329-334 (2007).

${ }^{9}$ C. Min, Z. Shen, J. Shen, Y. Zhang, H. Fang, G. Yuan, L. Du, S. Zhu, T. Lei, and X. Yuan, Nat. Commun. 4, 2891 (2013).

${ }^{10}$ D. Liu, B. Gu, B. Ren, C. Lu, J. He, Q. Zhan, and Y. Cui, J. Appl. Phys. 119(7), 073103 (2016).

${ }^{11}$ X. Wang, J. Ding, W. Ni, C. Guo, and H. Wang, Opt. Lett. 32(24), 3549-3551 (2007).
${ }^{12}$ S. Liu, P. Li, T. Peng, and J. Zhao, Opt. Express 20(19), 21715-21721 (2012).

${ }^{13}$ Z. Y. Rong, Y. J. Han, S. Z. Wang, and C. S. Guo, Opt. Express 22(2), 1636-1644 (2014).

${ }^{14}$ K. J. Moh, X. C. Yuan, J. Bu, D. K. Y. Low, and R. E. Burge, Appl. Phys. Lett. 89, 251114 (2006)

${ }^{15}$ X. Cai, J. Wang, M. J. Strain, B. Johnson-Morris, J. Zhu, M. Sorel, J. L. O'Brien, M. G. Thompson, and S. Yu, Science 338(6105), 363-365 (2012).

${ }^{16}$ D. Pohl, Appl. Phys. Lett. 20(7), 266 (1972).

${ }^{17}$ F. Enderli and T. Feurer, Opt. Lett. 34(13), 2030-2032 (2009).

${ }^{18}$ H. Ren, X. Li, Q. Zhang, and M. Gu, Science 352(6287), 805-809 (2016).

${ }^{19}$ L. Li, X. Zheng, C. Jin, M. Qi, X. Chen, Z. Ren, J. Bai, and Z. Sun, Appl. Phys. Lett. 105(22), 221103 (2014).

${ }^{20}$ D. Naidoo, F. S. Roux, A. Dudley, I. Litvin, B. Piccirillo, L. Marrucci, and A. Forbes, Nat. Photonics 10(5), 327-332 (2016).

${ }^{21}$ R. Zheng, C. Gu, A. Wang, L. Xu, and H. Ming, Opt. Express 18(10), 10834-10838 (2010).

${ }^{22}$ D. Lin and W. A. Clarkson, Opt. Lett. 40(4), 498-501 (2015).

${ }^{23}$ D. Lin, K. Xia, R. Li, X. Li, G. Li, K.-I. Ueda, and J. Li, Opt. Lett. 35(21), 3574-3576 (2010).

${ }^{24}$ S. Ramachandran, P. Kristensen, and M. F. Yan, Opt. Lett. 34(16), 2525-2527 (2009).

${ }^{25}$ T. Grosjean, D. Courjon, and M. Spajer, Opt. Commun. 203(1-2), 1-5 (2002).

${ }^{26}$ J. N. Blake, B. Y. Kim, H. E. Engan, and H. J. Shaw, Opt. Lett. 12(4), 281-283 (1987).

${ }^{27}$ W. Zhang, L. Huang, K. Wei, P. Li, B. Jiang, D. Mao, F. Gao, T. Mei, G. Zhang, and J. Zhao, Opt. Express 24(10), 10376-10384 (2016).

${ }^{28}$ B. Sun, A. Wang, L. Xu, C. Gu, Z. Lin, H. Ming, and Q. Zhan, Opt. Lett. 37(4), 464-467 (2012).

${ }^{29}$ J. Lin, K. Yan, Y. Zhou, L. X. Xu, C. Gu, and Q. W. Zhan, Appl. Phys. Lett. 107, 191108 (2015).

${ }^{30}$ B. Sun, A. Wang, C. Gu, G. Chen, L. Xu, D. Chung, and Q. Zhan, Opt. Lett. 40(8), 1691-1694 (2015).

${ }^{31}$ L. G. Wright, D. N. Christodoulides, and F. W. Wise, Nat. Photon. 9(5), 306-310 (2015).

${ }^{32}$ D. Mao, B. Du, D. Yang, S. Zhang, Y. Wang, W. Zhang, X. She, H. Cheng, H. Zeng, and J. Zhao, Small 12(11), 1489-1497 (2016).

${ }^{33}$ Z. Sun, T. Hasan, F. Torrisi, D. Popa, G. Privitera, F. Wang, F. Bonaccorso, D. M. Basko, and A. C. Ferrari, ACS Nano 4(2), 803-810 (2010).

${ }^{34}$ F. Wang, A. G. Rozhin, V. Scardaci, Z. Sun, F. Hennrich, I. H. White, W. I. Milne, and A. C. Ferrari, Nat. Nanotechnol. 3(12), 738-742 (2008).

${ }^{35}$ D. Mao, S. Zhang, Y. Wang, X. Gan, W. Zhang, T. Mei, Y. Wang, Y. Wang, H. Zeng, and J. Zhao, Opt. Express 23(21), 27509-27519 (2015).

${ }^{36}$ H. Yu, H. Zhang, Y. Wang, C. Zhao, B. Wang, S. Wen, H. Zhang, and J. Wang, Laser Photonics Rev. 7(6), L77-L83 (2013).

${ }^{37}$ Y. Xu, Z. Wang, Z. Guo, H. Huang, Q. Xiao, H. Zhang, and X.-F. Yu, Adv. Opt. Mater. 4(8), 1223-1229 (2016).

${ }^{38}$ M. Lipson, J. Lightwave Technol. 23(12), 4222-4238 (2005).

${ }^{39}$ T. Mizunami, T. V. Djambova, T. Niiho, and S. Gupta, J. Lightwave Technol. 18(2), 230-235 (2000).

${ }^{40}$ D. Mao, X. Liu, D. Han, and H. Lu, Opt. Lett. 38(16), 3190-3193 (2013).

${ }^{41}$ D. J. Richardson, J. M. Fini, and L. E. Nelson, Nat. Photonics 7(5), 354-362 (2013). 